\title{
Perfil de idosos admitidos em serviço de fisioterapia frente à sazonalidade
}

\author{
Profile of elderly admitted to a physical therapy center as a function of seasonality
}

\author{
Paulo Giusti Rossi ${ }^{1} \bowtie$, Ana Claudia Silva Farche ${ }^{1}$, Juliana Hotta Ansai ${ }^{1}$, Anielle Cristhine de Medeiros Takahashi ${ }^{1}$, \\ Marcello Ávila Mascarenhas ${ }^{2}$
}

1 Departamento de Fisioterapia, Programa de Pós-Graduação em Fisioterapia, Universidade Federal de São Carlos (UFSCAR). São Carlos, SP.

2 Programa de Pós-Graduação em Biociências e Reabilitação, Centro Universitário Metodista - IPA. Porto Alegre, RS.

\section{RESUMO}

OBJETIVOS: Analisar o perfil e a prevalência de doenças em idosos admitidos em duas estações do ano distintas, em um serviço público de fisioterapia de um município de médio porte localizado em zona de clima subtropical de altitude.

MÉTODOS: Um estudo transversal analisou prontuários de pacientes encaminhados nos meses que compreenderam o verão e o inverno de 2011. Foram incluídos os prontuários de idosos (acima de 60 anos de idade) de ambos os sexos e excluídos os prontuários sem informações sobre o diagnóstico médico atual. Foram coletadas as variáveis idade e sexo e os diagnósticos atuais e pregressos. Para análise comparativa quanto ao perfil dos idosos admitidos nos dois períodos do ano, utilizou-se o teste Qui-quadrado.

RESULTADOS: Foram analisados dados de 71 idosos. A média de idade foi de $69,2 \pm 8,1$ anos e $48(70,4 \%)$ eram do sexo feminino. Os diagnósticos mais frequentes no momento do encaminhamento foram de doenças traumato-ortopédicas $(60,6 \%)$. O maior número de admissões ocorreu durante o inverno $(62,0 \%)$. Os diagnósticos no momento do encaminhamento foram, respectivamente no verão e no inverno: traumatoortopédicos $17(63,0 \%)$ e $26(59,1 \%)$; reumatológicos $3(11,1 \%)$ e $10(22,7 \%)$; neurológicos $5(18,5 \%)$ e $4(9,1 \%)$; pós-operatório $2(7,4 \%)$ e $4(9,1 \%)(\mathrm{p}=0,64)$.

CONCLUSÕES: No inverno ocorreu maior demanda de encaminhamentos de idosos ao serviço de fisioterapia em comparação ao verão, com predomínio de mulheres em ambas as estações do ano. As causas de encaminhamento mais prevalentes foram relacionadas à traumatoortopedia, mas não houve diferença significativa na frequência dos diagnósticos conforme a estação do ano. O conhecimento sobre o perfil dos idosos admitidos em serviços de fisioterapia é importante para o planejamento de ações voltadas a essa faixa etária, com a realização de campanhas de informação, prevenção e promoção da saúde.

DESCRITORES: serviços de saúde comunitária; saúde do idoso; variações sazonais; fisioterapia.

\section{ABSTRACT}

AIMS: To analyze the profile and the prevalence of diseases in elderly patients admitted to a physical therapy center in a medium-sized Brazilian city located in a subtropical zone in two different seasons of the year.

METHODS: A cross-sectional study assessed the medical records of patients treated during summer and winter months in 2011. Medical records of male and female elderly patients (aged over 60 years) were included, and those without information on present medical diagnosis were excluded. Age and sex and both current and previous diagnostic records were analyzed. The chi-square test was used for the comparative analysis of the profile of the elderly patients for both seasons.

RESULTS: Seventy-one medical records were analyzed. The mean age was $69.2 \pm 8.1$ years and $48(70.4 \%)$ patients were female. There was a higher prevalence of trauma-orthopedic diseases $(60.6 \%)$. The greatest number of admissions was in winter $(62.0 \%)$. At the time of referral, there were 17 cases $(63.0 \%)$ of trauma-orthopaedic diseases in summer and $26(59.1 \%)$ in winter; 3 cases $(11.1 \%)$ of rheumatologic diseases in summer and $10(22.7 \%)$ in winter; 5 cases $(18.5 \%)$ of neurologic disorders in summer and $4(9.1 \%)$ in winter; and 2 cases $(7.4 \%)$ of postoperative complications in summer and $4(9.1 \%)$ in winter $(\mathrm{p}=0.64)$.

CONCLUSIONS: There was a greater demand for referrals of elderly patients to the physical therapy center in winter than in summer, with a predominance of female patients in both seasons. The most prevalent causes of referral were related to trauma-orthopaedic diseases, but there was no significant difference in the frequency of diagnoses according to season. Knowledge about the profile of the elderly admitted to physical therapy centers is important for targeting actions at this age group, conducting information, prevention, and health promotion campaigns.

KEY WORDS: community health services; health of the elderly; seasonal variations; physical therapy. 
Abreviatura: CMF, Centro Municipal de Fisioterapia.

\section{INTRODUÇÃO}

O acelerado envelhecimento populacional é uma realidade de âmbito mundial. De acordo com os últimos dados da Organização Mundial de Saúde, estima-se que em 2050 o número de indivíduos acima de 60 anos atinja dois bilhões [1]. No Brasil, o percentual de idosos atualmente corresponde a $13 \%$ da população total e as projeções indicam que esse valor chegará em $26 \%$ em 2060 [2]. Sob os pontos de vista físico e fisiológico, o envelhecimento caracteriza-se por uma degeneração gradual e progressiva dos órgãos, tecidos e metabolismo, acarretando a atenuação de suas funções [3]. Sabe-se que a debilidade do organismo no combate às enfermidades é acentuada pelos fatores sazonais, geralmente acarretando um aumento na procura por serviços de saúde durante os períodos frios do ano [3-7].

As doenças endêmicas dos idosos podem ser relacionadas com fatores geográficos. Essa população é mais vulnerável às mudanças climáticas, geologia e relevo, bem como à diminuição da qualidade do ar e às perturbações por eventos climáticos extremos [8-11]. Temperaturas extremamente altas provocam a incidência de choques térmicos, exaustão e câimbras pelo calor. Por outro lado, temperaturas extremamente baixas podem causar hipotermia e agravar males como artrite, sinusite, doenças respiratórias e enrijecimento das articulações [5-7, 10-12].

Um estudo avaliou o número de prescrições para idosos em uma unidade de saúde da França durante o inverno e o verão e observou que as prescrições de medicamentos para os sistemas cardiovascular e respiratório aumentaram significativamente nos meses de temperaturas mais baixas [6]. Ainda, outros autores observaram que o número de idosos admitidos no setor público de urgência e emergência por complicações respiratórias aumenta durante o inverno [7]. É importante analisar a relação entre os efeitos das variações climáticas e doenças nos idosos em cada região, para que planos e ações sejam implementados de forma mais adequada, levando em consideração as variações sazonais [3].

Um dos recursos terapêuticos utilizados pelos idosos para assegurar a manutenção da capacidade funcional é a fisioterapia. As técnicas fisioterapêuticas têm papel importante na preservação da função física dos idosos, com o intuito de prevenir ou adiar a instalação de incapacidades, diminuindo assim o comprometimento imposto por enfermidades incapacitantes e promovendo uma melhor adaptação do indivíduo à sua realidade $[13,14]$.

Assim, este estudo teve como objetivo analisar o perfil e a prevalência de doenças em idosos admitidos em duas estações do ano distintas, em um serviço público de fisioterapia de um município de médio porte localizado no sul do Brasil.

\section{MÉTODOS}

Realizou-se um estudo transversal, cujo projeto foi aprovado pelo Comitê de Ética em Pesquisa do Centro Universitário Metodista IPA, sob número 296/2011, seguido da aquiescência da Secretaria Municipal de Saúde de um município situado no Sul do Brasil, assim como do respectivo Centro Municipal de Fisioterapia (CMF).

O CMF é um serviço de atenção secundária à saúde, vinculado ao Sistema Único de Saúde, que serve de referência para os pacientes atendidos na atenção primária. O CMF recebe encaminhamentos de diferentes faixas etárias, desde crianças até idosos. Quando encaminhado, o paciente pode ser admitido imediatamente ou permanecer em lista de espera, dependendo da situação clínica, até ser chamado via contato telefônico para atendimento. A prioridade do atendimento dá-se frente à complexidade do caso encaminhado.

A amostra do estudo foi constituída por idosos cadastrados no CMF, que estavam em atendimento, já haviam sido atendidos ou estavam na lista de espera. Consideraram-se dois grupos conforme o período de admissão no serviço no ano de 2011: Grupo 1, encaminhado de janeiro a março (verão); e Grupo 2, encaminhado de julho a setembro (inverno). Foram incluídos no estudo indivíduos de ambos os sexos com idade igual ou superior a 60 anos. Os dados foram obtidos dos prontuários, sendo excluídos os prontuários que não apresentavam informações sobre o diagnóstico médico atual. As variáveis do estudo foram idade, sexo e diagnósticos atuais relacionados aos motivos do encaminhamento/admissão no serviço, além dos diagnósticos pregressos, conforme a descrição médica presente nos prontuários. As informações referentes aos diagnósticos foram agrupadas conforme a especialidade clínica correspondente.

Para análise estatística utilizou-se o programa IBM SPSS versão 21.0, e nível de significância $\alpha=0,05$. Foi realizada uma análise descritiva dos dados e analisadas as diferenças quanto ao perfil de idosos entre os dois períodos do ano pelo teste Qui-quadrado. 


\section{RESULTADOS}

A partir da análise dos prontuários, foram localizados 78 pacientes que atingiam os critérios de inclusão. Destes, foram excluídos sete prontuários por não possuírem diagnóstico médico descrito no encaminhamento, resultando em 71 idosos incluídos no estudo. Verificou-se que $44(62,0 \%)$ admissões no CMF ocorreram no período do inverno.

Com relação às variáveis sociodemográficas, as idades variaram entre 60 e 100 anos, com média de $69,2 \pm 8,1$ anos; e $48(67,6 \%)$ idosos eram do sexo feminino. Ao comparar o perfil dos idosos entre os dois períodos de admissão, não houve diferença significativa quanto à idade e ao sexo. Houve uma porcentagem maior de homens no Grupo 2, encaminhados no inverno ( $\mathrm{n}=18,40,9 \%$ ) com relação ao Grupo 1 , encaminhados no verão $(n=5,18,5 \%)$, porém essa diferença não foi significativa (Tabela 1).

Tabela 1. Dados sociodemográficos de 71 idosos encaminhados em duas estações do ano distintas a um serviço público de fisioterapia de um município de médio porte localizado em zona de clima subtropical de altitude.

\begin{tabular}{lcccc}
\hline \multicolumn{1}{c}{$\begin{array}{c}\text { Variáveis } \\
\mathbf{N}(\%)\end{array}$} & $\begin{array}{c}\text { Grupo 1 } \\
\mathbf{n = 2 7} \\
\mathbf{n}(\%)\end{array}$ & $\begin{array}{c}\text { Grupo 2 } \\
\mathbf{n = 4 4} \\
\mathbf{n}(\%)\end{array}$ & Total & $\mathbf{p}^{*}$ \\
Sexo & $22(45,8 \%)$ & $26(54,2 \%)$ & 48 & 0,068 \\
Feminino & $5(21,7 \%)$ & $18(78,3 \%)$ & 23 & \\
Masculino & & & & \\
Faixa Etária & $15(35,7 \%)$ & $27(64,3 \%)$ & 42 & \\
60 a 69 anos & $9(49,0 \%)$ & $13(59,1 \%)$ & 22 & 0,936 \\
70 a 79 anos & $3(42,9 \%)$ & $4(57,1 \%)$ & 7 & \\
80 anos ou mais & &
\end{tabular}

Grupo 1: idosos encaminhados no verão; Grupo 2: idosos encaminhados no inverno. * Teste Qui-quadrado.
Verificaram-se 29 diagnósticos distintos de acordo com as descrições médicas nos prontuários. Os diagnósticos mais frequentes foram ruptura de manguito rotador com 12 casos $(15,2 \%)$, seguido de causas neurológicas com $9(11,4 \%)$ casos e dor lombar com $6(7,6 \%)$ casos. As informações referentes aos 71 diagnósticos atuais e sete diagnósticos pregressos foram agrupadas conforme a especialidade clínica encontrada (traumato-ortopedia, reumatologia, neurologia e pós-operatório) Com relação aos encaminhamentos por especialidade, a traumato-ortopedia apresentouse em maior número em ambos os grupos, com 17 $(63,0 \%)$ casos no verão e $26(59,1 \%)$ no inverno, seguidos de reumatologia e neurologia. Quando avaliados os diagnósticos encaminhados e agrupados por especialidade clínica, verificou-se que não foram encontradas diferenças estatisticamente significativas com relação aos dois períodos do ano (Tabela 2).

Quanto aos dados referentes aos diagnósticos pregressos, apenas três prontuários continham essas informações preenchidas. Osteoporose, doença de Parkinson, dor no ombro, dificuldade no sono, osteoartrose de joelho, osteoartrose de coluna lombar e lesão ligamentar do tornozelo foram os diagnósticos pregressos encontrados na pesquisa.

\section{DISCUSSÃO}

O presente estudo analisou o perfil da população idosa usuária do CMF de um município de médio porte do sul do Brasil, encaminhada para atendimento em dois períodos do ano distintos. Verificou-se que o maior número de admissões foi durante o inverno e os diagnósticos mais prevalentes foram os relacionados à traumato-ortopedia.

Tabela 2. Diagnóstico/especialidade informados por 71 idosos encaminhados em duas estações do ano distintas a um serviço público de fisioterapia de um município de médio porte localizado em zona de clima subtropical de altitude.

\begin{tabular}{|c|c|c|c|c|}
\hline Especialidade médica & $\begin{array}{c}\text { Grupo } 1 \\
n=27 \\
n(\%)\end{array}$ & $\begin{array}{c}\text { Grupo } 2 \\
n=44 \\
n(\%)\end{array}$ & $\begin{array}{l}\text { Total } \\
\text { n (\%) }\end{array}$ & $\mathbf{p}^{*}$ \\
\hline \multicolumn{5}{|l|}{ Diagnóstico atual } \\
\hline Traumato-ortopedia & $17(63,0)$ & $26(59,1)$ & $43(60,6)$ & \multirow{4}{*}{0,64} \\
\hline Reumatologia & $3(11,1)$ & $10(22,7)$ & $13(18,3)$ & \\
\hline Neurologia & $5(18,5)$ & $4(9,1)$ & $9(12,7)$ & \\
\hline Pós-operatório, sem informação da especialidade & $2(7,4)$ & $4(9,1)$ & $6(8,5)$ & \\
\hline \multicolumn{5}{|l|}{ Diagnóstico pregresso } \\
\hline Traumato-ortopedia & $1(16,7)$ & $1(100,0)$ & $2(28,6)$ & \multirow{3}{*}{1,00} \\
\hline Reumatologia & $3(50,0)$ & $0(0)$ & $3(42,8)$ & \\
\hline Neurologia & $2(33,3)$ & $0(0)$ & $2(28,6)$ & \\
\hline
\end{tabular}

Grupo 1: idosos encaminhados no verão, Grupo 2: idosos encaminhados no inverno.

* Teste Qui-quadrado. 
Os dados referentes à faixa etária assemelham-se aos encontrados no estudo de Cardoso e Costa [15], o qual objetivou traçar o perfil epidemiológico de idosos associados em planos de saúde, incluindo na amostra apenas usuários de planos de saúde privados, ao contrário do presente estudo. No Brasil, no ano de 2005, os atendimentos ambulatoriais de fisioterapia pelo Sistema Único de Saúde foram responsáveis por gastos públicos de cerca de 122 milhões de reais ao ano [16]. Em um estudo recente desenvolvido em um ambulatório de fisioterapia, observou-se que os idosos admitidos apresentavam idade média de 73,7 anos e a doença associada mais frequente foi o acidente vascular cerebral. Além disso, o sedentarismo, a dificuldade de deambular, o etilismo e o histórico de tabagismo são mais associados ao sexo masculino, enquanto que depressão, dores crônicas, prática de atividade física e osteoporose ao feminino [17].

O município no qual o presente estudo foi realizado tem predomínio do clima subtropical de altitude, que se caracteriza por inverno seco, com temperaturas médias mínimas de $8^{\circ} \mathrm{C}$ e verão ameno, com médias máximas de $26^{\circ} \mathrm{C}$ [18]. Estudos prévios observaram que as variações térmicas podem influenciar o aumento da morbidade e de hospitalizações em indivíduos acima de 60 anos $[9,10]$, especialmente em baixas temperaturas [5, 7]. Esses achados concordam com o presente estudo, que detectou maior porcentagem de encaminhamentos nos meses de baixa temperatura. Talvez a diferença não tenha sido maior em razão das diferenças de temperatura não serem tão acentuadas quanto em países do hemisfério norte.

Neste estudo foi possível identificar que os encaminhamentos de idosos do sexo feminino foram os mais prevalentes nos dois períodos. O predomínio feminino está de acordo com o padrão demográfico brasileiro atual e pelo fato das mulheres procurarem mais serviços de saúde quando comparadas aos homens $[2,19]$. Identificou-se também um percentual maior de idosos do sexo masculino nos meses de inverno em relação aos meses de verão, embora sem significância estatística, o que pode ser justificado pela pequena amostragem.

Nesse contexto, o impacto da baixa temperatura merece atenção para que os serviços de saúde estejam preparados para atender uma demanda maior de indivíduos no inverno, especialmente em regiões de alterações climáticas acentuadas. Além disso, alguns autores mostram que as baixas temperaturas agravam as doenças e dores crônicas [5-7, 13], reduzem a qualidade de vida [11] e diminuem o nível de atividade física e a adesão de idosos a programas de tratamentos [20,21]. Com isso, destaca-se a importância de programas de promoção, prevenção e controle de doenças crônicas, bem como inclusão de fatores motivacionais nessa época do ano.

Os dados relacionados à prevalência de diagnósticos por especialidades mostram a traumatoortopedia, a reumatologia e a neurologia como as especialidades com encaminhamentos mais frequentes. A prevalência de encaminhamentos de idosos com patologias traumato-ortopédicas no inverno também é justificada pelo clima frio, pois a hipotermia está ligada a mecanismos de enrijecimento articular e muscular, o que leva a doenças reumáticas e ortopédicas [3].

Destaca-se como limitação do estudo a escassez de informações de dados nos cadastros e prontuários bem como a ausência de diagnósticos médicos, o que limitou a descrição do perfil dos idosos usuários no CMF. Dessa maneira, deve-se observar que as taxas de encaminhamento não refletem necessariamente a incidência ou a prevalência dessas morbidades na população idosa em geral. Ainda, a análise intergrupo ficou restrita devido ao pequeno tamanho amostral.

Conclui-se que no inverno ocorreu maior demanda de encaminhamentos de idosos ao serviço de fisioterapia em relação ao verão, com predomínio de mulheres nas duas estações do ano. As causas de encaminhamento mais prevalentes foram relacionadas à traumatoortopedia, mas não houve diferença significativa na frequência dos diagnósticos conforme a estação do ano. O perfil dos idosos admitidos em serviços de fisioterapia é importante para o planejamento de ações voltadas a essa faixa etária, com a realização de campanhas de informação, prevenção e promoção da saúde.

\section{NOTAS}

Apoio financeiro

Este estudo não recebeu apoio financeiro de fontes externas.

\section{Declaração de conflito de interesses}

Os autores declaram não haver conflitos de interesses relevantes ao conteúdo deste estudo, informam ter tido acesso a todos os dados obtidos e assumem completa responsabilidade pela integridade dos resultados. 


\section{REFERÊNCIAS}

1. Organização Mundial da Saúde. OMS. "Ageing well" must be a global priority [Internet]. 2015 [cited 2015 Sept 28]. Available from: http://www.who.int/mediacentre/news/releases/2014/lancet-ageing-series/en/

2. Instituto Brasileiro de Geografia e Estatística. Projeção da População do Brasil por Sexo e Idade para o Período 2000/2060 [Internet]. 2013 [cited 2015 Nov 3]. Avaiable from: <http://www.ibge.gov.br>.

3. Nogueira VBM, Nogueira RN, Cândido GA, de Souza VC, da Silva SSF. Efeitos das alterações climáticas e antrópicas na saúde do idoso. RBCEH. 2011;8(1):88-106.

4. Shoemaker MJ, Roper SE, Calkins TE. Seasonal variation of daily physical activity in individuals with heart failure. Heart Int. 2016;11(1): 25-31. https://doi.org/10.5301/heartint.5000231

5. Alahmari AD, Mackay AJ, Patel AR, Kowlessar BS, Singh R, Brill SE, Allinson JP, Wedzicha JA, Donaldson GC. Influence of weather and atmospheric pollution on physical activity in patients with COPD. Respir Res. 2015 June 13;16:71. https://doi.org/10.1186/ s12931-015-0229-Z

6. Durant R, Boulanger-Morel M, Ferry J, Blain A, Blain H, Jeandel C. Drug prescriptions for the elderly. Results of a survey on 8,809 prescriptions obtained by coding in the North-East area of France. Presse Med. 2003 Apr 12;32(14):630-7.

7. Bellali H, Zaghouani R, Alaya NB, Chahed MK. Emergency department visits for respiratory symptoms in greater Tunisia, 2007 and 2010. Tunis Med. 2015 July;93(7):465-9.

8. Brandon CA, Gill DP, Speechley M, Gilliland J, Jones GR. Physical activity levels of older community dwelling adults are influenced by summer weather variables. Appl Physiol Nutr Metab. 2009 Apr;34(2):182-90. https://doi.org/10.1139/H09-004

9. Carneseca EC, Achcar JA, Martinez EZ, Godini J, Hotta LK. Contagem diária de hospitalizações e variações climáticas da cidade de São Paulo: uma abordagem Bayesiana. Rev Bras Biom. 2010;28(1):57-72.

10. Alonso JB, Achcar JA, Hotta LK. Climate changes and their effects in the public health: use of poisson regression models. Pesqui Oper. 2010;30(2):427-42. https://doi.org/10.1590/S0101-74382010000200010

11. Nakao M, Yamauchi K, Ishihara Y, Solongo B, Ichinnorov D. Effects of air pollution and seasonality on the respiratory symptoms and health-related quality of life (HR-QoL) of outpatients with chronic respiratory disease in Ulaanbaatar: pilot study for the comparison of the cold and warm seasons. Springerplus. 2016 Oct 19;5(1):1817. eCollection 2016.

12. Ayoade JO. Introdução à climatologia para trópicos. Rio de Janeiro: Bertrand Brasil; 2004.

13. Knorst MR, Sousa MAT, Bós AJG. Necessidades fisioterapêuticas de idosos em atendimento ambulatorial. RBCEH. 2010;7(1):11-21. https://doi.org/10.5335/rbceh.2010.002

14. Duarte FM, Araújo KA, Oliveira ES, Lima MPSS. A importância da fisioterapia na promoção da qualidade de vida para os idosos. CCBS. 2013;1:1-9.

15. Cardoso JH, Costa JSD. Características epidemiológicas, capacidade funcional e fatores associados em idosos de um plano de saúde. Ciênc Saúde Coletiva. 2010;15(6):2871-8. https://doi.org/10.1590/S1413-81232010000600024

16. Plentz RDM. Gasto governamental do SUS com a fisioterapia no Brasil. Rev Bras Fisioter. 2006;10(2):777-8.

17. Melo-Neto JS, Mendes AP, de Aragão IG, Alves AS, Corrêa PR, Romano EM. Perfil dos pacientes atendidos no Setor de Fisioterapia Cardiorrespiratória de uma clínica escola de São José do Rio Preto - SP. Arq Ciênc Saúde. 2012;19(4)108-13.

18. Instituto Nacional de Meteorologia (BR). Normais Climatológicas do Brasil 1961-1990 [Internet]. 2015 [cited 2015 Aug 19]. Available from: http://www.inmet.gov.br/

19. Louvison MCP, Lebrão ML, de Oliveira Duarte YA, Laurenti R. Desigualdades nas condições de saúde e no uso de serviços entre as pessoas idosas do município de São Paulo: uma análise de gênero e renda. Saúde Colet. 2008;5(24):189-94.

20. Klenk J, Büchele G, Rapp K, Franke S, Peter R; ActiFE Study Group. Walking on sunshine: effect of weather conditions on physical activity in older people. J Epidemiol Community Health. 2012 May;66(5):474-6. https://doi.org/10.1136/jech.2010.128090

21. Witham MD, Donnan PT, Vadiveloo T, Sniehotta FF, Crombie IK, Feng Z, McMurdo ME. Association of day length and weather conditions with physical activity levels in older community dwelling people. PLoS One. 2014 Jan 30;9(1):e85331. https://doi. org/10.1371/journal.pone.0085331 